\title{
Teil II:
}

Unterbringung von Flüchtenden mit besonderen Schutzbedarfen 


\section{„Don't let your past determine your future.” Erfahrungsbericht einer geflüchteten Frau ${ }^{1}$}

Layla Asisa ${ }^{2}$

\section{Einfübrung}

Hallo! Ich bin Layla Asisa.

Ich arbeite und lebe in Berlin. Seit September 2016 bin ich in Deutschland. Ich bin mit meinem Exfreund gekommen. Aber ich war allein in Berlin. Er wurde in eine andere Stadt weit entfernt geschickt und ich bin in Berlin allein geblieben, weil wir nicht verheiratet waren.

\section{In der Sammelunterkunft}

Offiziell war die Unterkunft, in der ich untergebracht war, eine besondere Unterkunft für Frauen. Praktisch war aber alles schlecht. Es gab nur männliche Sicherheitsdienstmitarbeiter und die meisten Sozialarbeiter waren ebenfalls männlich und es gab immer Stress. Am Anfang wohnten für zwei oder drei Monate auch Männer in der Unterkunft. Dann blieben nur Minderjährige bis 17 Jahre gemeinsam mit ihren Müttern dort wohnen. Aber auch weiterhin waren der Sicherheitsdienst und auch die Sozialarbeiter männlich. Es war immer sehr dreckig. Für die Zimmer eines Flurs gab es nur eine Toilette und keine, absolut keine hygienischen Maßnahmen. Immer wieder gab es Krankheiten und Infektionen. Niemand hat uns darüber informiert. Wir haben nur davon erfahren, als wir den Sicherheitsdienst Masken tragen sahen. Es gab eine Gruppe von Frauen, die hatten eine Infektion. Vielleicht weil sie immer in einem Raum zusammen aßen. Der ganze Sicherheitsdienst hatte Masken an, aber niemand hat uns etwas gesagt. Die Frauen haben auch im selben Raum das Essen bekommen wie

1 Wir haben Layla Asisa im Frühsommer 2020 getroffen und sie hat uns von ihrem Leben in Deutschland, insbesondere von ihren Erfahrungen in den verschiedenen Unterkünften und ihrem Engagement in der Monitoring Group erzählt. Wir haben das Interview anschließend zu einem Text zusammengefasst.

2 Dieser Name ist ein Pseudonym, das die Autorin gewählt hat. 
wir. Und niemand hat uns etwas gesagt. Wir wissen, dass es eine Infektion war, weil die anderen das erzählt haben. Aber wir wissen nicht, welche Krankheit sie hatten und warum sie sie nicht in Quarantäne geschickt haben oder so was.

Ich war in dieser Unterkunft bis Juni 2017. Wir waren manchmal zwei, manchmal drei in einem Zimmer. Das hing davon ab, wie groß das Zimmer ist. Es gab keine Küche. Das Essen war immer sehr dreckig und verschimmelt, man konnte kaum erkennen, was es eigentlich war. Die ganze Unterkunft hat gestunken, das hat man sogar von der Straße aus gerochen. Ich erinnere mich daran, dass wir einmal so einen kleinen Protest gemacht haben. Wir haben gesagt: Das Essen ist schlecht! Daraufhin haben sie uns alle bedroht und die Frauen irgendwo anders hingeschickt und nichts hat sich verändert.

In der Unterkunft war es ein bisschen lächerlich, weil der Sicherheitsdienst überall hin durfte. Als Ausrede benutzen sie, dass die anderen Minderjährigen die kleinen Kinder vergewaltigen könnten und sie das überprüfen müssten. Ich bin einmal mein Gesicht waschen gegangen und als ich wieder hochkam, standen hinter mir zwei Männer. Ein anderes Mal war ich im Speisessaal und wollte zur Toilette gehen und meine Hände waschen. Einer der Männer war hinter mir. Sie hatten mich zur Toilette verfolgt. Das Problem in der Flüchtlingsunterkunft ist das, dass du zwar die Duschkabine zumachen kannst, aber die Tür ist sehr kurz. Zwischen Boden und Tür gibt es Platz und auch oben gibt es Platz. Das war schrecklich, immer Angst zu haben. Es war auch so dreckig. Du versuchst deinen Körper sauber zu machen und dann ist da einfach Dreck. So, wie sagt man, eine Sauerei.

Manche Frauen werden vom Leben in einer Flüchtlingsunterkunft traumatisiert. Ich habe das vielleicht geschafft, weil ich ein bisschen stärker bin. Wenn du dein Kind baden willst und du gehst zu so einer dreckigen Toilette, und es gibt keine Seife. Wir haben uns immer wieder beschwert, dass es keine Seife und kein Desinfektionsmittel gibt. Die Frauen, die so leben, werden traumatisiert, auch die Männer. Leute, die kein Englisch sprechen, nur Afghanisch oder Arabisch oder so, sie sind einfach gefangen. Sie können nicht raus aus der Situation. Sobald man eine eigene Wohnung hat, verbessert sich das Leben richtig, das habe ich auch bei anderen Frauen gesehen. Auch wenn es nur eine kleine Wohnung ist. Dann kannst du zumindest lernen und kontrollieren, was dein Kind macht oder nicht macht, isst oder nicht isst.

In der Flüchtlingsunterkunft habe ich mit meinen eigenen Augen gesehen, wie ein Mädchen auf die Toilette gegangen ist und kleine Jungs, sechs bis acht oder neun Jahre alt, haben die Türe aufgemacht und standen 
um sie herum. Sie war auf der Toilette mit der Hose unten. Was ist das für ein Leben?! Und als die Frau sich beschwert hat, haben sie sie in eine Turnhalle verlegt. Eine kurdische Frau, eine Jesidin, eine besonders schutzbedürftige Frau. Sie ist nicht einfach so hierhergekommen. Sie hatte tatsächlich ein krasses Problem in ihrem Land, weißt du. Niemand sollte sie so behandeln.

Es gibt auch sehr gute Unterkünfte, aber manchmal stehen sie leer. In anderen Unterkünften werden die Frauen gestaut. Den Grund dafür kenne ich nicht. Ich war auch schon zu Besuch bei Leuten, die in besseren Unterkünften leben. Dort ist es fast wie ein normales Leben.

In meiner Unterkunft gab es dann einen Vorfall mit einem männlichen Sicherheitsdienstmitarbeiter. Dann habe ich mich beim Sozialarbeiter beschwert, dann wurde es aber nur schlimmer. Sie haben alle anderen vom Sicherheitsdienst informiert und das war nicht anonym. Ich habe das Problem auch beim LAF $^{3}$ geschildert, dass ich in Gefahr bin und mich nicht sicher fühle. Wir durften die Tür nicht abschließen. Der Mitarbeiter im LAF hat dazu gesagt: „Ja, wenn dir das nicht gefällt, könnte ich dich in eine Sporthalle schicken, wo es keine Trennung zwischen Betten von Männern und Frauen gibt." Dann hat eine Deutschlehrerin mir geholfen, einen Platz in einem Frauenhaus zu bekommen. Das ist ein normales Frauenhaus, aber geflüchtete Frauen dürfen dort auch hingehen.

\section{Im Frauenhaus, in einer Schutzwohnung und in einer eigenen Wohnung}

Im Frauenhaus waren immer drei Frauen in einem Zimmer unterbracht. Das war auch nicht traumhaft. Ich erinnere mich, dass die Toilette und die Dusche kein Schloss hatten, dass die Tür immer auf sein musste. Das verstehe ich nicht. Das Problem war, es gab eine Frau, die hat die ganze Zeit andere Frauen angefasst. Und dann haben wir uns bei der Betreuerin beschwert. Ich hatte Angstanfälle und ich hatte Alpträume. Die Frau hat einmal zu uns gesagt: „Ich werde euch alle vergewaltigen.“ Dann haben wir mit der Sozialarbeiterin geredet und sie hat gesagt, sie hat mit der Frau geredet und hat sie gewarnt. Okay, sie kann mögen, wen sie will, aber sie darf nicht mit den Frauen so reden, sie bedrohen oder zu etwas zwingen. Die Frau hat danach damit aufgehört.

Im Frauenhaus habe ich meine Gesamtleistung vom LAF bekommen. Ich habe mir Essen gekauft. In jedem Flur gab es eine Küche, die sich sechs

3 Landesamt für Flüchtlingsangelegenheiten. 
Frauen geteilt haben. Das war besser, nicht so schlimm. Schwierig waren nur die Sozialhelferinnen. Nach sechs Monaten hat uns die Betreuerin Bescheid gegeben, dass sie die Unterkunft räumen wollen, da sie eine neue Unterkunft für das Frauenhaus bekommen. Sie wollten einfach jede Frau von uns in irgendeine Flüchtlingsunterkunft, auch gemischte, schicken. Sie hätten uns helfen müssen, eine Wohnung zu bekommen oder einen Platz in einem anderen Frauenhaus. Aber sie wollten uns einfach rausschmeißen, alle. Das war sehr schlimm.

Ich erinnere mich daran, dass es eine russische Frau gab mit Kindern. Sie hatte ein Problem mit ihrem Mann, er wollte sie töten. Die Betreuerin wollte die Frau von ihren Kindern trennen. Die Frau sollte allein in eine Flüchtlingsunterkunft, in ein Zimmer mit anderen Frauen. Die Kinder wollte sie zu ihrem Vater schicken. Aber dann hat die Frau Hilfe gekriegt von einer Familienhelferin vom Jugendamt. Die hat ihr sehr gut geholfen. Die Kinder blieben bei ihr und sie hat jetzt eine tolle Flüchtlingsunterkunft. Das ist eine bessere Unterkunft, wo sie zwei Zimmer mit Toilette und Küche allein hat. Die auch nicht weit weg von der Schule ihrer Kinder ist.

Ich habe den Betreuerinnen dann gesagt, dass ich nicht in einer Flüchtlingsunterkunft wohnen kann, wo auch Männer leben oder es männliches Sicherheitspersonal gibt. Ich bin traumatisiert von den damaligen Ereignissen, ich kann das nicht noch einmal machen. Dann hat eine von denen mir eine Liste mit Schutzwohnungen und Telefonnummern gegeben, wo ich selbst anrufen sollte. Ich habe überall angerufen. Ich habe dann sehr nette Menschen von einer Initiative kennengelernt. Sie waren sehr nett und haben mir geholfen. Sie haben mir ein Zimmer in einer WG für Frauen gegeben. Das war eine ganz normale Schutzwohnung. Das ist die nächste Stufe nach dem Frauenhaus. Denn ein Frauenhaus ist nur eine akute Lösung.

Wir waren eine Frau und eine Frau mit Kind. Die Wohnung war immer sauber. Sie haben sich um uns gekümmert, uns dabei unterstützt, einen Lebenslauf zu schreiben und mich für einen Job zu bewerben. Sie waren sehr, sehr nett. Das war sehr toll. Sie haben mir ebenfalls geholfen, meine Wohnung zu bekommen. Sie wollten mich auch unterstützen, gebrauchte Möbel zu bekommen. Sie sind auch bis heute sehr hilfreich und sehr nett.

Im Juni 2018 habe ich die Wohnung gefunden, in der ich jetzt noch wohne. Sie haben mir dabei geholfen. Wir haben viele Bewerbungen für Wohnungen geschrieben. Dann haben wir diese Wohnung gefunden. 


\section{Auf der Suche nach einer Community und Gleichgesinnten: Die Monitoring Group in der Zukunftswerkstatt}

Ich habe mich ziemlich einsam in der Sammelunterkunft, in der ich zuerst gewohnt habe, gefühlt. Aber dann habe ich Menschen gesucht, die ehrenamtlich aktiv sind, eine Community oder so. Ich habe mich in unterschiedlichen Communities ehrenamtlich engagiert. Bei der Monitoring Group in der Zukunftswerkstatt bin ich schließlich geblieben. Das Monitoring-Projekt ${ }^{4}$ hat mich mehr interessiert. Ehrenamtlich engagiere ich mich in der Zukunftswerkstatt mit dem Monitoring-Projekt seit 2016.

Ich war erst bei einer anderen Frauengruppe aktiv. Sie kamen im September zu uns, um vor unserer Flüchtlingsunterkunft Flyer zu verteilen. Ich wollte mich gerne in einer Community von Frauen engagieren, damit wir uns gegenseitig unterstützen und reden, auch einfach, dass ich nicht allein bin. Einmal haben sie gesagt, dass es ein Treffen mit dem Staatssekretär für Integration geben soll. Wir sind dann dorthin gegangen, um sie zu unterstützen. Dort habe ich Frauen von der Monitoring Group kennengelernt. Sie haben mich immer wieder zu Workshops für Frauen eingeladen. Es gab viele Workshops, über Empowerment für Frauen, rechtliche Workshops mit Rechtsanwälten, Sozialberatung, manchmal auch ärztliche Beratung. Sie haben z.B. eine Frauenärztin eingeladen. Sie hat auch erzählt, was man machen kann, wenn eine Frau schwanger ist oder Schmerzen hat. Es gab unterschiedliche Sachen, das war echt toll. Sie haben mich auch zu Treffen und Veranstaltungen eingeladen, wo viele Frauen zusammenkommen und sich über ihre Erfahrungen austauschen und wir gemeinsam überlegen, was wir machen können. Das war sehr toll. Ich wusste immer, was die nächsten Schritte sind. Z.B. habe ich auch Einladungen zum Flüchtlingsrat bekommen. Einmal habe ich drei Tage bei einem Workshop in Brandenburg mitgemacht.

Der Grund, warum ich bei der Zukunftswerkstatt geblieben bin, ist, dass es auch in ehrenamtlichen Initiativen Diskriminierung gibt. Eine solche Erfahrung hatte ich in einer anderen Initiative von Frauen aus mittelund südafrikanischen Ländern gemacht. Dort haben sie mich immer als weißen Menschen gesehen und mich richtig diskriminiert. Das war ein bisschen verwirrend für mich: Auf der Straße werde ich bespuckt, weil ein

4 Im Rahmen des Monitoring-Projekts wurde ein unabhängiges Beschwerdemanagement für geflüchtete Menschen in Unterkünften von geflüchteten Frauen und Unterstützter*innen entwickelt. Vgl. dazu auch den Beitrag von Rajaa Al Khlefawi, Namarek Al Shallal und Annika Khan in diesem Band. 
deutscher Mensch denkt, dass ich schwarz bin. Aber manche afrikanischen Leute denken, dass ich weiß bin und sie diskriminieren mich. Ich habe eine Hautfarbe dazwischen, ich bin nicht weiß und nicht schwarz. Aber das Problem ist die Wahrnehmung der anderen.

Wir wurden von der Zukunftswerkstatt unterstützt, wenn wir z.B. Probleme mit dem LAF hatten oder eine Frage bei der Wohnungssuche. Aber wir machen auch etwas für die Gesellschaft. Wir gehen z.B. in den Nachbarschaftsgarten in Pankow und pflegen die Pflanzen oder wir helfen bei Veranstaltungen, z.B. beim Weihnachtsmarkt, da unterstützen wir die deutsche Nachbarschaft. Ich finde das ist echt toll. Das ist echte Integration. Auch kochen und essen wir zusammen. Beim Marathon laufen Deutsche und Menschen mit Migrationsgeschichte zusammen mit. Man fühlt sich nicht so, auf Englisch gesagt, „alienated“. Weil, wenn jemand dich isoliert oder du dich isolierst, dann gehörst du nicht dazu, das ist das Problem.

Es gibt viele Menschen mit Migrationsgeschichte, die dazugehören wollen. Ich bin z.B. aus meinem Heimatland gegangen, nicht wegen Krieg, sondern weil ich nicht dazu gehörte und ich nicht akzeptiert wurde. Es ging so weit, dass es für mich dort lebensbedrohlich war. Ich wollte in Deutschland eine Community, eine Gruppe finden, die mich akzeptiert, wie ich bin. Und auch ich möchte die anderen akzeptieren, wie sie sind. Ich möchte mit leben und nicht die ganze Zeit in Isolation sein und denken, dass die deutsche Gesellschaft eine bösartige sei, die uns diskriminiert. Ich wurde auch in meinem Heimatland diskriminiert, jeden Tag, als Frau. Das passiert in Deutschland ab und zu auch. Ich will nicht rechnen wievielmal mir das passiert. Im Vergleich mit dem, was mir in mein Heimatland passiert, ist das vielleicht 10\%. Das ist in meinem Fall. Vielleicht ist es im Fall von jemandem anderen hier keine tolle Situation. Aber ich, ich finde es toll.

Deswegen bin ich gerne bei der Zukunftswerkstatt, weil wir zusammen und integriert sind und jeder die anderen akzeptiert. Niemand sagt, du musst dich anpassen, du musst das machen. Wenn ich was mache, mach ich es, weil ich es gerne mache. Manche Frauen tragen ein Kopftuch und sie werden auch akzeptiert. Sie werden immer herzlich willkommen geheißen, sie werden immer unterstützt. Ich habe auch gespürt, dass mein Leben und das der anderen Frauen besser geworden ist, weil wir einander unterstützen. Z.B. unterstütze ich immer noch ehrenamtlich andere Frauen beim Schriftverkehr, wenn sie Post von der Bank oder der Schule bekommen und noch kein Deutsch können. Sie schicken mir dann eine WhatsApp-Nachricht, und ich helfe ihnen dann. 
Seit Corona haben sich ein paar Sachen in unserer Arbeit in der $\mathrm{Zu}-$ kunftswerkstatt geändert. Wir haben jetzt eine WhatsApp-Gruppe, in welcher ich z.B. die aktuellen Corona-Regelungen aus der Zeitung oder aus den Nachrichten in einfaches Arabisch übersetze. Denn manche Frauen denken immer noch, dass sie zuhause bleiben müssten. Wir erzählen von den Lockerungen, z.B. dass die Schulen wieder auf sind oder dass es die Maskenpflicht gibt. Aus den Unterkünften höre ich nichts mehr, da die Frauen nicht mit mir reden dürfen. Die erleben Druck der Sozialarbeiter, diese haben wirklich Macht.

\section{Abschluss}

Für mich hat sich die Situation seit Corona nicht viel verändert. Ich habe eine kleine Wohnung und bin froh über die Privatsphäre. Die Nachbarn sind sehr nett. Ich kann mich an die Regeln halten, weil ich Deutsch lesen kann. Ich schaue Nachrichten und informiere mich darüber, was man machen darf und was nicht. Ich habe gehört, dass manche Kurzarbeit bekommen haben. Bei meiner Arbeit machen wir Projekte im Öffentlichkeitsbereich, also mit der Regierung. Die Firma hat daher keine Auftragsprobleme, im Gegenteil, sie haben mehrere Leute neu angestellt. Sie brauchen Mitarbeiter.

Übrigens, ich habe meine Arbeit durch die Zukunftswerkstatt bekommen. 
